\title{
The prevalence and clinical characteristics of anemia in Korean patients with inflammatory bowel disease
}

\author{
Dae Sung Lee, Ki Bae Bang, Ji Yeon Kim, Yoon Suk Jung, Jung Ho Park, Hong Joo Kim, Yong Kyun Cho, \\ Chong Il Sohn, Woo Kyu Jeon, Byung Ik Kim, Kyu Young Choi, Dong Il Park \\ Department of Internal Medicine, Sungkyunkwan University School of Medicine, Kangbuk Samsung Hospital, Seoul, Korea
}

\begin{abstract}
Background/Aims: Quality of life is closely related to anemia in patients with inflammatory bowel disease (IBD). Several studies have reported on anemia in patients with IBD in Western countries. This study investigated the prevalence and clinical characteristics of anemia in Korean patients with IBD. Methods: We reviewed the medical records of 92 patients with ulcerative colitis (UC) and 76 patients with Crohn's disease (CD) who were followed regularly at a single tertiary medical center in Korea between January 2003 and December 2012. Hemoglobin (Hb) thresholds used to define anemia were $<13.0 \mathrm{~g} / \mathrm{dL}$ in men and $<12.0 \mathrm{~g} / \mathrm{dL}$ in women according to the World Health Organization criteria. We chose the lowest Hb level in each year as a representative value because $\mathrm{Hb}$ levels changed at each examination and anemia was associated with disease deterioration. The relationship between clinical variables and lowest $\mathrm{Hb}$ level was assessed. Results: The prevalence of anemia was $36.3 \%$ in patients with UC and $41.6 \%$ in patients with CD. Anemia in patients with CD was associated with hospital admission, 5-aminosalicylate (5-ASA) and infliximab treatment in men. Anemia in patients with UC was associated with hospital admission, oral steroid use, thiopurine and infliximab treatment in men. Conclusions: The prevalence of anemia in Korean patients with IBD was comparable to that of patients in Western countries. Anemia was associated with male patients with CD who were admitted to the hospital and received medications including 5-ASA and infliximab, and men with UC who were admitted to the hospital and received medications including oral steroids, thiopurine and infliximab. (Intest Res 2016;14:43-49)
\end{abstract}

Key Words: Crohn disease; Colitis, ulcerative; Anemia

\section{INTRODUCTION}

The prevalence of anemia is higher in patients with IBD than in the general population of Western countries. ${ }^{1-3}$ However, the prevalence of anemia with IBD patients is unknown in the Korean population. A recent cohort study from Norway reported that the prevalence of anemia in patients with IBD was higher than that in the general population, and anemia was more common in patients with CD than in UC. ${ }^{1}$

Received December 3, 2014. Revised May 11, 2015.

Accepted May 29, 2015.

Correspondence to Dong II Park, Department of Internal Medicine, Kangbuk

Samsung Hospital, Sungkyunkwan University School of Medicine, 29

Saemunan-ro, Jongno-gu, Seoul 03181, Korea. Tel: +82-2-2001-8555, Fax:

+82-2-2001-8360, E-mail: diksmc.park@samsung.com

Financial support: None. Conflict of interest: None.
Anemia in IBD has received little attention compared to other extra-intestinal complications. Clinicians have often overlooked the clinical importance of anemia in patients with IBD, because of the perception that anemia in patients with IBD is a common finding and an inevitable result of the disease course. However, anemia is well known to be a condition that is related to patient quality of life (QOL) and ability to work. ${ }^{2,4}$ Several studies have revealed that the correction of anemia has significantly benefited patients with IBD in terms of QOL and ability to work.

Because there are many causes of anemia in patients with IBD, various approaches are needed to diagnose and treat it. ${ }^{7,8}$ Anemia may be caused by chronic blood loss from inflammation and ulceration, folate deficiency, malnutrition, hemolysis, and marrow-suppressing medication. In addition, chronic abdominal pain and nausea often result in poor oral

๑ Copyright 2016. Korean Association for the Study of Intestinal Diseases. All rights reserved.

This is an Open Access article distributed under the terms of the Creative Commons Attribution Non-Commercial License (http://creativecommons.org/licenses/by-nc/4.0)

which permits unrestricted non-commercial use, distribution, and reproduction in any medium, provided the original work is properly cited. 
intake, and mucosal inflammation in the gastrointestinal tract may lead to inadequate nutrient absorption. Anemia of chronic disease, described as a nonspecific consequence of inflammatory cytokine network activation, results from ineffective erythropoiesis and shortened red blood cell (RBC) survival. ${ }^{9,10}$

If the disease worsens, the prevalence of anemia increases. In these patients, vigorous medical treatment of the underlying disease will decrease the severity of anemia. However, despite this occasionally anemia does not improve in patients with IBD in clinical and/or endoscopic remission.

The aim of this study is to investigate the prevalence, type, natural course, time to recovery, and risk factors for anemia in Korean patients with IBD.

\section{METHODS}

\section{Patients}

This was a retrospective cohort study performed in a single tertiary medical center in Korea between January 2003 and December 2012. Institutional review board approval for the current study was obtained from Kangbuk Samsung Hospital.

The diagnoses of UC and CD were confirmed by previously established international criteria based on clinical, endoscopic, histopathological, and radiological findings. ${ }^{11}$ The definitions and diagnosis of CD and UC were based on the European Crohn's and Colitis Organization consensus documents. ${ }^{12,13}$ Specifically, the definitions of CD and UC are found in the revised Montreal classification, in which the clinical patterns of IBD are further characterized. ${ }^{14,15}$

We reviewed electronic medical records, including demographic data and endoscopic and radiologic examinations, to define the extent of disease, ascertain the medications used, and determine the results of the actual laboratory tests at the time of the first diagnosis and every year after diagnosis in patients with UC and CD who were followed regularly for more than a year. We performed routine laboratory, endoscopic and radiologic examinations on all patients who had been diagnosed with IBD at the first evaluation. Additional examinations were done after symptom flare-up to check for: (1) the appearance of typical symptoms of UC or $\mathrm{CD}$, such as diarrhea, abdominal pain, weight loss, perianal fistula, perianal abscess, or rectal bleeding; (2) the receipt of a new prescription or increasing dose of immunosuppressant, corticosteroid, cyclosporine, or infliximab in existing medications; and (3) the incidences of hospitalization or surgery due to the worsening of symptoms or the develop- ment of complications. We did not conduct anemia studies on all patients with anemia in this study.

Children aged $<19$ years, pregnant women, patients followed for less than a year, and patients who had diseases that are known to cause anemia such as chronic kidney disease, gastrectomy, and hematologic disease were excluded. Demographics, clinical data, endoscopic activity, and clinical outcomes were collected from the medical records.

\section{Definition of Anemia}

Hemoglobin $(\mathrm{Hb})$ concentration is the most reliable factor for the diagnosis of anemia. Measuring $\mathrm{Hb}$ concentration is relatively easy and inexpensive, and this measurement is frequently used as a surrogate marker of anemia. However, if the only indicator of anemia used is Hb concentration, then determination of the etiology of anemia should be performed with caution. Hb distributions differ with the sex, age, regional origin, and ethnicity of the patient as well as the patient's physiological status; for example, $\mathrm{Hb}$ concentrations may differ when the patient has chronic kidney disease or hematologic disease, has recently undergone chemotherapy or is pregnant. ${ }^{16}$ In this study, World Health Organization (WHO) $\mathrm{Hb}$ thresholds were used to classify individuals living at sea level as anemic. ${ }^{16,17}$ The WHO defines anemia as a $\mathrm{Hb}$ level of $<13.0 \mathrm{~g} / \mathrm{dL}$ in men and $<12.0 \mathrm{~g} / \mathrm{dL}$ in women. We chose the lowest level each year as the representative concentration of the year, because $\mathrm{Hb}$ concentrations changed at each examination and the lowest $\mathrm{Hb}$ concentration represents the deterioration of IBD.

\section{Statistical Analysis}

Data were collected and entered into Microsoft Excel 2010 (Microsoft Corp., Redmond, WA, USA). The software program SPSS version 18.0 (SPSS Inc., Chicago, IL, USA) was used for statistical analyses. The chi-square or Fisher's exact test was used to compare categorical variables. The Student's $t$-test or the Mann-Whitney $U$ test was used to compare numerical variables between the groups. $P$-values of $<0.05$ were considered statistically significant. Adjusted ORs and 95\% CIs were calculated.

\section{RESULTS}

\section{Patient Demographics}

Of the 187 patients with IBD, 19 were excluded for the 
Table 1. Baseline Characteristics of Ulcerative Colitis Patients

\begin{tabular}{|c|c|c|c|c|c|c|}
\hline \multirow{2}{*}{ Variable } & \multicolumn{3}{|c|}{ Female $(n=37)$} & \multicolumn{3}{|c|}{ Male $(n=55)$} \\
\hline & Anemic & Non anemic & $P$-value & Anemic & Non anemic & $P$-value \\
\hline Hospitalization & $10(27.0)$ & $6(16.2)$ & 0.269 & $13(23.6)$ & $15(27.2)$ & 0.017 \\
\hline Total bowel resection & $4(10.8)$ & $3(8.1)$ & 0.669 & $8(14.5)$ & $13(23.6)$ & 0.056 \\
\hline 5-ASA & $20(54.0)$ & $12(32.4)$ & 1.000 & $19(34.5)$ & $30(54.5)$ & 0.674 \\
\hline Oral steroid & $9(24.3)$ & $5(13.5)$ & 1.000 & $10(18.1)$ & $3(5.4)$ & 0.003 \\
\hline IV steroid & $2(5.4)$ & $1(2.7)$ & 1.000 & $2(3.6)$ & 0 & 0.156 \\
\hline Thiopurine & $8(21.6)$ & $1(2.7)$ & 0.112 & $10(18.1)$ & $3(5.4)$ & 0.003 \\
\hline Infliximab & 0 & 0 & & $5(9.0)$ & 0 & 0.008 \\
\hline Disease extent & & & 0.075 & & & 0.544 \\
\hline Proctitis & $14(37.8)$ & $13(35.1)$ & & $16(29.0)$ & $25(45.4)$ & \\
\hline Left-sided & $4(10.8)$ & $1(2.7)$ & & $2(3.6)$ & $5(9.0)$ & \\
\hline Extensive disease & $5(13.5)$ & 0 & & $4(7.2)$ & $3(5.4)$ & \\
\hline
\end{tabular}

Values are presented as $n(\%)$.

5-ASA, 5-aminosalicylate; IV, intravenous.

Table 2. Baseline Characteristics in Crohn's disease Patients

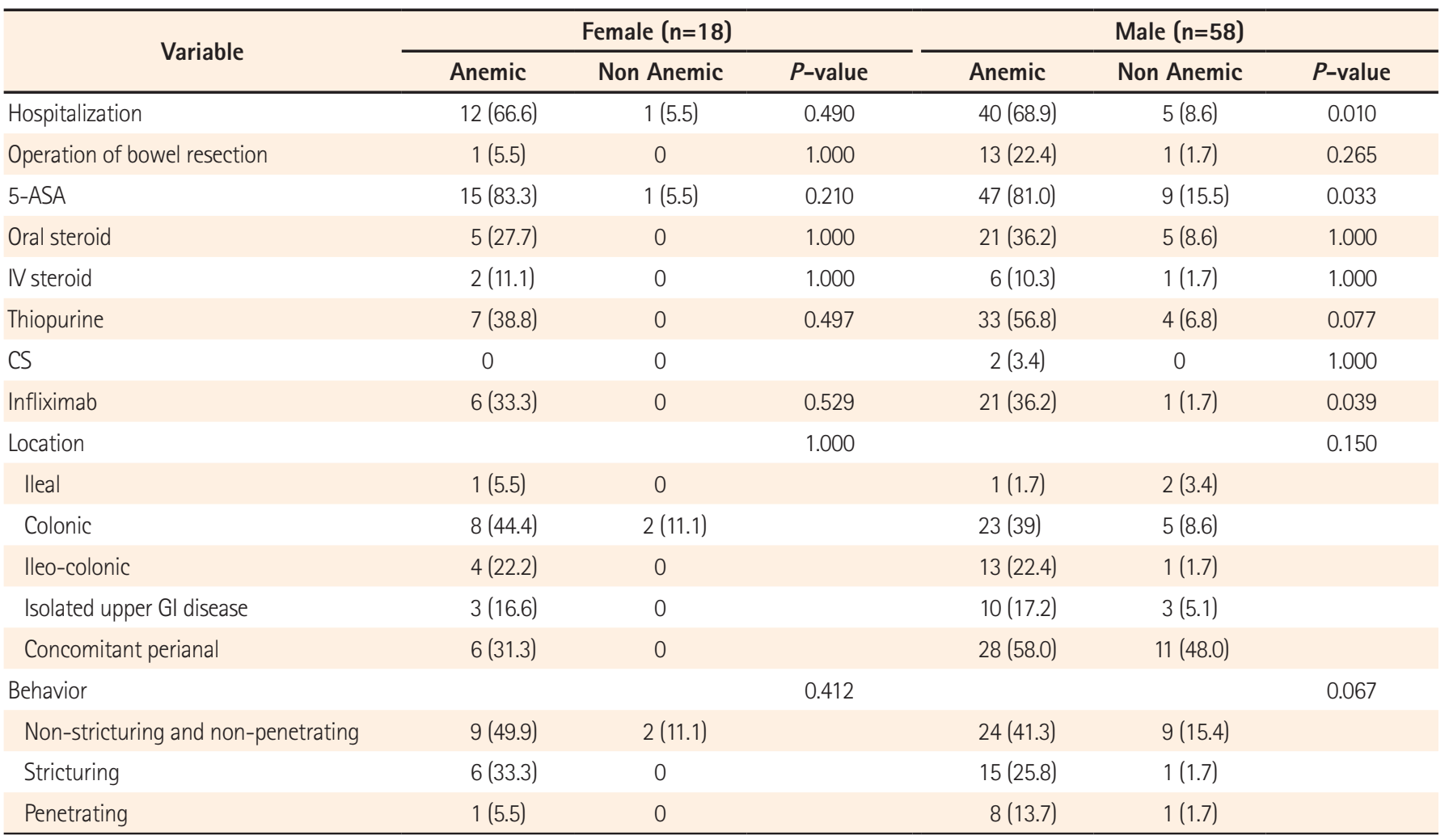

Values are presented as $n(\%)$.

5-ASA, 5-aminosalicylate; IV, intravenous; CS, cycloserine; GI, gastrointestinal.

following reasons: less than a year of follow-up $(n=7)$ and incomplete clinical data $(\mathrm{n}=12)$. Finally, 168 patients (92 patients with UC and 76 patients with $\mathrm{CD}$ ) were included in the study. Demographic and clinical characteristics of patients with IBD are presented in Table 1 and Table 2. The clinical behaviors and severity are classified by the revised Montreal 

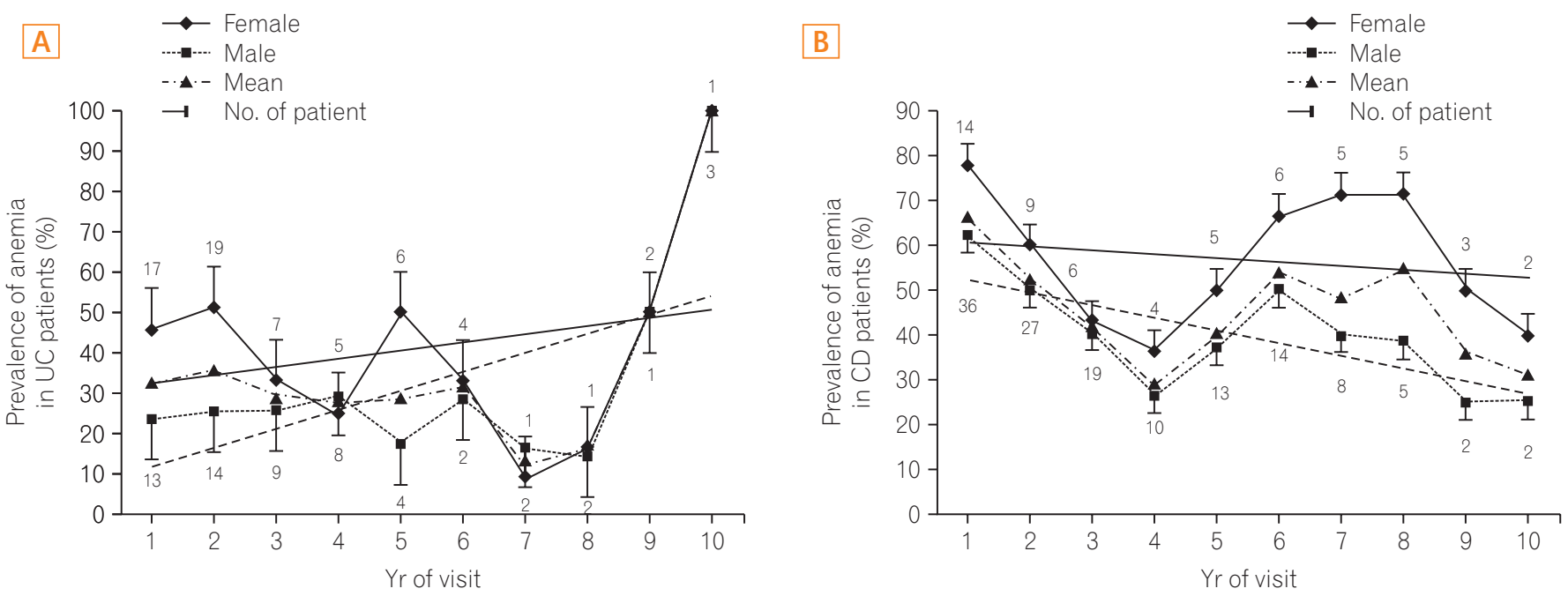

Fig. 1. Prevalence of anemia in patients with IBD. Mean prevalence of anemia in patients with IBD is higher than that of the general population. Anemia is more common in patients with CD than those with UC. There was a larger variation in the prevalence of anemia in patients with $C D$ than those with UC. (A) The prevalence of anemia is higher in women with UC than in men with UC (41.4\% in women and $33.1 \%$ in men). (B) The prevalence of anemia is higher in women with $C D$ than in men with $C D$ (56.6\% in women and $39.4 \%$ in men).

Table 3. Classification of Anemia Type by Mean Cell Volume and Mean Concentration of Hemoglobin per Volume of Red Cells

\begin{tabular}{lccc}
\hline \multicolumn{1}{c}{ Type } & Hypochromic & Normochromic & Hyperchromic \\
\hline Microcytic & 1 & 2 & 3 \\
Normochromic & 4 & 5 & 6 \\
Macrocytic & 7 & 8 & 9 \\
\hline
\end{tabular}

classification, in which the clinical patterns of IBD are fully characterized. The mean age at diagnosis in patients with CD was younger than that in patients with UC (39.8 years vs. 53.3 years, $P<0.001$ ). The mean follow-up time (disease duration) of patients with IBD was not significantly different (UC was 55.6 months and CD was 51.9 months, $P=0.440$ ).

\section{Prevalence of Anemia}

The mean prevalence of anemia was $36.3 \%$ in patients with UC (41.4\% in women and $33.1 \%$ in men) (Fig. 1A). The mean prevalence of anemia was $41.6 \%$ in patients with CD (56.6\% in women and $39.4 \%$ in men). The prevalence of anemia in patients with UC decreased gradually over the 8 years of follow-up visits. The prevalence of anemia fluctuated and then decreased in patients with CD (Fig. 1B).

\section{Type of Anemia}

We divided the type of anemia according to the red cell indices (Table 3) of the mean cell volume and mean concen-
Table 4. Type of Anemia by Red Cell Indices

\begin{tabular}{cccccc}
\hline & \multicolumn{2}{c}{ CD } & & \multicolumn{2}{c}{ UC } \\
\cline { 2 - 3 } \cline { 5 - 6 } Type & $\begin{array}{c}\text { Female } \\
(\mathbf{n = 1 0 1 )}\end{array}$ & $\begin{array}{c}\text { Male } \\
(\mathbf{n}=\mathbf{2 9 8})\end{array}$ & & $\begin{array}{c}\text { Female } \\
(\mathbf{n}=160)\end{array}$ & $\begin{array}{c}\text { Male } \\
(\mathbf{n}=\mathbf{2 1 3})\end{array}$ \\
\hline 1 & $9(8.9)$ & $23(7.7)$ & $13(8.1)$ & $13(6.1)$ \\
2 & $1(1.0)$ & 0 & $2(1.3)$ & $4(1.9)$ \\
3 & 0 & 0 & 0 & 0 \\
4 & $17(16.8)$ & $35(11.7)$ & $11(6.8)$ & $7(3.3)$ \\
5 & $66(65.3)$ & $192(64.4)$ & $115(71.9)$ & $169(79.3)$ \\
6 & $4(4.0)$ & $35(11.7)$ & $14(8.8)$ & $12(5.6)$ \\
7 & $1(1.0)$ & $1(0.3)$ & 0 & 0 \\
8 & $3(3.0)$ & $10(3.4)$ & $3(1.9)$ & $7(3.3)$ \\
9 & 0 & $2(0.7)$ & $2(1.3)$ & $1(0.5)$ \\
\hline
\end{tabular}

Values are presented as $n(\%)$.

tration of $\mathrm{Hb}$ per volume of red cells. Normocytic normochromic anemia was the most common type of anemia in patients with CD (65.3\% in women and $64.4 \%$ in men) and in patients with UC (71.9\% in women and $79.3 \%$ in men) (Table 4$){ }^{17}$

\section{Pattern of Hb Level}

Hb levels tended to rise slowly in UC except in the 6th year of the follow-up visits. In women with UC, the highest level was $12.8 \mathrm{~g} / \mathrm{mL}$, which occurred during the 7th year, and the lowest level was $10.5 \mathrm{~g} / \mathrm{mL}$, which occurred during the $1 \mathrm{st}$ 

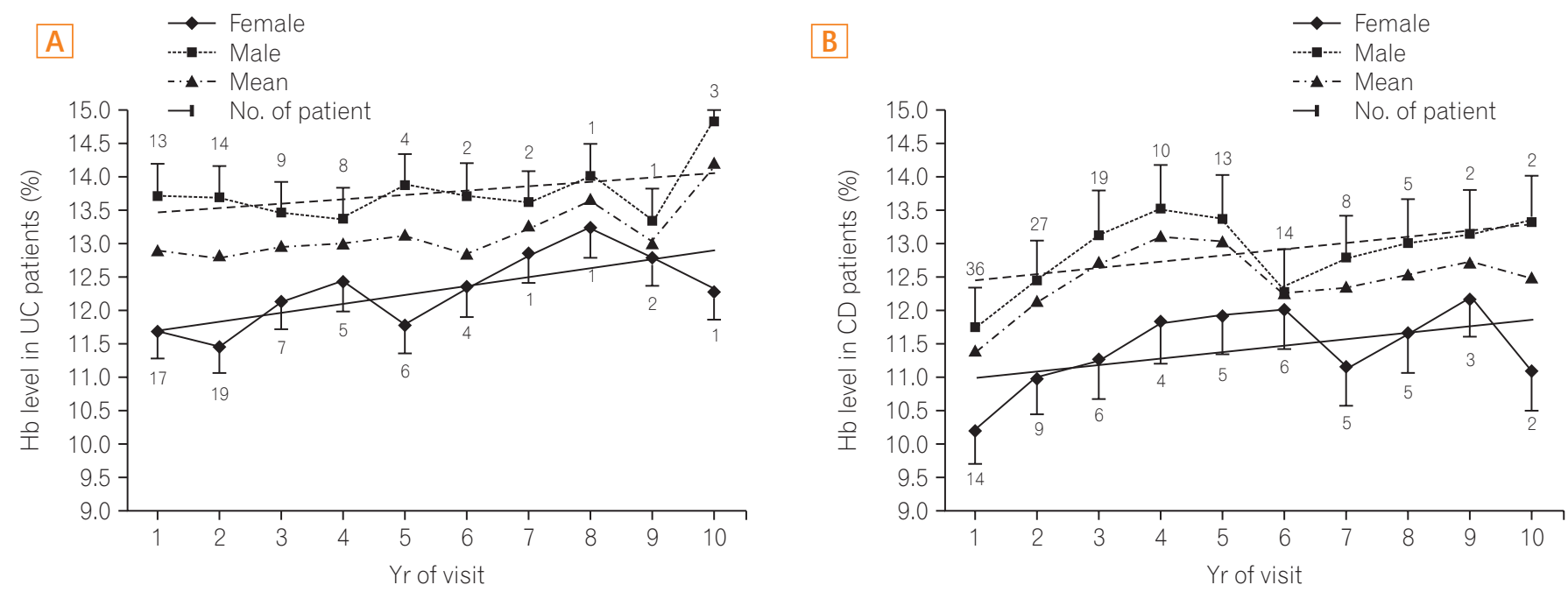

Fig. 2. Hemoglobin ( $\mathrm{Hb}$ ) level in IBD patients. The mean $\mathrm{Hb}$ level is higher in patients with UC than in patients with CD. (A) Male patients with UC are not anemic during the follow up period, while female patients with UC are not anemic during the second half of a 10-years follow up period. (B) CD patients are anemic during most time of follow period. This anemia is more severe in females with CD than in males with CD.

year of visits. In men with UC, the average level was $>13.0 \mathrm{~g} /$ $\mathrm{mL}$ during the 10 years of follow-up (Fig. 2A).

Levels in patients with CD tended to rise until the 4th year of visits and decreased during the 5th and 6th years of visits. In women with $\mathrm{CD}$, the highest level was $12.2 \mathrm{~g} / \mathrm{mL}$, found in the 9th year of visits, and the lowest level was $10.2 \mathrm{~g} / \mathrm{mL}$, in the 1st year of visits. In men with CD, the highest level was $13.5 \mathrm{~g} / \mathrm{mL}$, in the 4 th year of visits, and the lowest level was $11.7 \mathrm{~g} / \mathrm{mL}$, in the 1st year of visits (Fig. 2B).

Differences in $\mathrm{Hb}$ between the maximum and minimum levels were of a larger range in patients with $\mathrm{CD}$ than in patients with UC ( $1.8 \mathrm{~g} / \mathrm{mL}$ vs. $0.3 \mathrm{~g} / \mathrm{mL}$, respectively) (Fig. 2).

\section{Factors Related to Anemia}

When anemia was detected, the factors which were associated with anemia were analyzed for each year. Anemia was associated with hospitalization $(P=0.017)$ and oral steroid $(P=0.003)$, as well as thiopurine $(P=0.003)$ and infliximab $(P=0.008)$ treatment in men with UC (Table 1). Anemia was associated with hospitalization $(P=0.010)$ and 5-aminosalicylate $(P=0.033)$ and infliximab $(P=0.039)$ treatment in men with CD (Table 2). However, there was no significant association between anemia and clinical characteristics such as admission, surgery, medications, and disease extent in women with UC or CD.

\section{DISCUSSION}

The prevalence of anemia in Korean patients with IBD was similar to that found in the Western population. ${ }^{1-3}$ Wilson et al. reported a systematic review of the literature of anemia in IBD patients. ${ }^{10}$ Prevalence varies by age (adults or children) and patient population (ambulatory or hospitalized). Overall, the estimated prevalence ranged from $8.8 \%$ to $73.7 \%$ : the prevalence in ambulatory patients ranged from $8.8 \%$ to $72.7 \%$, and in hospitalized patients, the range was from $31.7 \%$ to $73.7 \%$. The prevalence of anemia ranged from $10.2 \%$ to $72.7 \%$ in patients with $\mathrm{CD}$, from $8.8 \%$ to $66.6 \%$ in patients with UC, and from $17.5 \%$ to $73.7 \%$ in patients with undifferentiated IBD. ${ }^{10}$ Harries et al. studied the association between anemia and CD in 55 ambulatory patients, reporting that a total of $43.6 \%$ patients were anemic. ${ }^{18}$ This result was similar to the prevalence of anemia in CD patients in our study. Schreiber et al. reported that the prevalence of anemia in patients with UC was $36.8 \%$, which was similar to the prevalence of anemia in our study. ${ }^{19}$

The most common type of anemia in this study was normocytic normochromic anemia. Anemia was associated with admission and 5-aminosalicylate and infliximab treatment in male patients with CD. In addition, anemia was associated with admission and oral steroid, thiopurine, and infliximab treatment in male patients with UC. However, there was no significant association between anemia and clinical characteristics such as admission, surgery, medications, and disease extent in women with UC and CD. To our 
knowledge, this is the first study that investigated the prevalence, type, natural course, time to recovery, and risk factors for anemia in Korean patients with IBD.

Disease severity may influence the prevalence of anemia in men with IBD because hospital admission and oral steroid, thiopurine, and infliximab use showed an association with anemia. Significant physiologic blood loss such as that which occurs during menstruation may affect the association between anemia and disease severity in women with IBD. None of the factors were found to be statistically significant in female patients. The small number of female patients compared with male patients may have contributed to this result. In this study, a high prevalence of anemia was present at the time of diagnosis, especially in the CD group (56.6\%); however, the prevalence decreased significantly over the follow-up period. This could have been affected by vigorous treatment of the underlying disease and the provision of iron supplementation. The decreasing prevalence of anemia is more notable in patients with CD than in patients with UC because medical treatment may decrease the severity of inflammation in the small bowel, including the duodenum where iron is absorbed by facilitated diffusion in patients with CD. The prevalence of anemia in UC patients soared to $50 \%-100 \%$ during the last two years of visits (Fig. 1A). There were only a small number of patients that were included in the last two years of visits; hence, missing data may make assessment of the actual prevalence during the last two years of visits difficult.

The prevalence of anemia decreased after the 1st year of visits; however, it did not decrease continuously over the 10 years of follow-up. Previous cohort studies have shown that a large proportion of patients enter remission as time progresses. ${ }^{20,21}$ However, the prevalence of anemia was not shown to decrease steadily. Insufficient recovery from anemia despite vigorous medical treatment may be explained partially by the fact that the most common type of anemia found in this study was normocytic normochromic anemia. Normocytic normochromic anemia is related to hypoproliferation of RBC. The majority of hypoproliferative anemia is caused by marrow damage and inadequate erythropoietin stimulation by inflammatory cytokines. ${ }^{22}$ Thiopurine could influence the occurrence of normocytic normochromic anemia because azathioprine induces megaloblastic anemia. Microcytic anemia by iron deficiencycombined with megaloblastic anemia by thiopurine can result in normocytic normochromic anemia. Therefore, patients who take thiopurine can show a combination of macrocytic and microcytic RBC. ${ }^{23}$

Our study had several limitations. First, we did not evalu- ate iron profiles to differentiate the cause of anemia. We only used red cell indices (mean corpuscular volume and mean corpuscular concentration). Second, we did not evaluate the correlation between the Hb level and clinical or biochemical disease activity indices. Third, we did not have information about iron supplementation. Oral or intravenous iron supplementation may contribute to the declining prevalence of anemia in patients with IBD. ${ }^{24,25}$ Fourth, a short follow-up period restricted the stratified analysis and multiple regression analysis. Fifth, we did not check for QOL in this study.

In summary, the prevalence of anemia at different points during the course of disease in Korean patients with IBD was similar to the prevalence of anemia in the Western population. The prevalence of anemia decreased after the 1st year of visits; however, it did not decrease continuously during the 10 years of follow-up. The awareness of the impact of anemia in this cohort seemed to be lower than expected, often leading to an inconsequent diagnostic approach and even a lack of treatment. Therefore, special attention is needed to improve the quality of care, especially with respect to improving anemia in patients with IBD.

\section{REFERENCES}

1. Høivik ML, Reinisch W, Cvancarova M, Moum B; IBSEN study group. Anaemia in inflammatory bowel disease: a populationbased 10-year follow-up. Aliment Pharmacol Ther 2014;39:69-76.

2. Pizzi LT, Weston CM, Goldfarb NI, et al. Impact of chronic conditions on quality of life in patients with inflammatory bowel disease. Inflamm Bowel Dis 2006;12:47-52.

3. Kim YJ. Intravenous iron and oral iron treatment in anemia of inflammatory bowel disease. Intest Res 2013;11:227-228.

4. Wells CW, Lewis S, Barton JR, Corbett S. Effects of changes in hemoglobin level on quality of life and cognitive function in inflammatory bowel disease patients. Inflamm Bowel Dis 2006; 12:123-130

5. Evans RW, Rader B, Manninen DL. The quality of life of hemodialysis recipients treated with recombinant human erythropoietin. Cooperative Multicenter EPO Clinical Trial Group. JAMA 1990;263:825-830.

6. Gasché C, Dejaco C, Waldhoer T, et al. Intravenous iron and erythropoietin for anemia associated with Crohn disease. A randomized, controlled trial. Ann Intern Med 1997;126:782787.

7. Auerbach M. Oral or IV iron in inflammatory bowel disease. Am J Gastroenterol 2012;107:950; author reply 950-951. 
8. Gomollón F, Gisbert JP. Current management of iron deficiency anemia in inflammatory bowel diseases: a practical guide. Drugs 2013;73:1761-1770.

9. Cronin CC, Shanahan F. Anemia in patients with chronic inflammatory bowel disease. Am J Gastroenterol 2001;96:2296-2298.

10. Wilson A, Reyes E, Ofman J. Prevalence and outcomes of anemia in inflammatory bowel disease: a systematic review of the literature. Am J Med 2004;116(Suppl 7A):44S-49S.

11. Lennard-Jones JE. Classification of inflammatory bowel disease. Scand J Gastroenterol Suppl 1989;170:2-6; discussion 16-19.

12. Dignass A, Lindsay JO, Sturm A, et al. Second European evidence-based consensus on the diagnosis and management of ulcerative colitis part 2: current management. J Crohns Colitis 2012;6:991-1030.

13. Dignass A, Van Assche G, Lindsay JO, et al. The second European evidence-based Consensus on the diagnosis and management of Crohn's disease: Current management. J Crohns Colitis 2010;4:28-62.

14. Satsangi J, Silverberg MS, Vermeire S, Colombel JF. The Montreal classification of inflammatory bowel disease: controversies, consensus, and implications. Gut 2006;55:749-753.

15. Silverberg MS, Satsangi J, Ahmad T, et al. Toward an integrated clinical, molecular and serological classification of inflammatory bowel disease: report of a Working Party of the 2005 Montreal World Congress of Gastroenterology. Can J Gastroenterol 2005;19(Suppl A):5A-36A.

16. Koller $\mathrm{O}$. The clinical significance of hemodilution during pregnancy. Obstet Gynecol Surv 1982;37:649-652.
17. McLean E, Cogswell M, Egli I, Wojdyla D, de Benoist B. Worldwide prevalence of anaemia, WHO Vitamin and Mineral Nutrition Information System, 1993-2005. Public Health Nutr 2009; 12:444-454

18. Harries AD, Fitzsimons E, Dew MJ, Heatley RV, Rhodes J. Association between iron deficiency anaemia and mid-arm circumference in Crohn's disease. Hum Nutr Clin Nutr 1984;38:47-53.

19. Schreiber S, Howaldt S, Schnoor M, et al. Recombinant erythropoietin for the treatment of anemia in inflammatory bowel disease. N Engl J Med 1996;334:619-623.

20. Solberg IC, Lygren I, Jahnsen J, et al. Clinical course during the first 10 years of ulcerative colitis: results from a populationbased inception cohort (IBSEN Study). Scand J Gastroenterol 2009;44:431-440.

21. Solberg IC, Vatn MH, Høie O, et al. Clinical course in Crohn's disease: results of a Norwegian population-based ten-year follow-up study. Clin Gastroenterol Hepatol 2007;5:1430-1438.

22. Spivak JL. Iron and the anemia of chronic disease: vindication for the non-essential role of iron supplementation. Oncology (Williston Park) 2011;25:421-423.

23. Gearry RB, Barclay ML. Azathioprine and 6-mercaptopurine pharmacogenetics and metabolite monitoring in inflammatory bowel disease. J Gastroenterol Hepatol 2005;20:1149-1157.

24. Avni T, Bieber A, Steinmetz T, Leibovici L, Gafter-Gvili A. Treatment of anemia in inflammatory bowel disease-systematic review and meta-analysis. PLoS One 2013;8:e75540.

25. Lugg S, Beal F, Nightingale P, Bhala N, Iqbal T. Iron treatment and inflammatory bowel disease: what happens in real practice? J Crohns Colitis 2014;8:876-880. 